\title{
DA DETECÇÃO DE SINAIS DE RISCO PARA AUTISMO À INTERVENÇÃO PRECOCE
}

\author{
FROM THE DETECTION OF INDICATORS OF RISK FOR AUTISM \\ TO EARLY INTERVENTION
}

\author{
DE LA DETECCIÓN DE SEÑALES DE RIESGO PARA AUTISMO \\ A LA INTERVENCIÓN
}

Odila Maria Ferreira de Carvalho Mansur ${ }^{1}$, Leila Regina d'Oliveira de Paula Nunes ${ }^{2}$

\section{RESUMO}

O Transtorno do Espectro Autista (TEA) é caracterizado por déficits persistentes na comunicação e interação social, padrões de comportamentos repetitivos e interesses restritos. O objetivo deste estudo foi verificar o efeito da aplicação de um programa de ensino por pais/cuidadores sobre o desempenho de seu filho. Participaram uma menina de 18 meses, com TEA, e sua cuidadora. Trata-se de pesquisa quase experimental, intrassujeitos, do tipo AB (linha de base, tratamento e follow-up). Foi conduzido um programa individualizado para ensinar a cuidadora a utilizar procedimentos lúdicos, visando promover a interação social e o aumento de turnos interacionais de ambos os participantes. Os comportamentos de ambos os participantes foram registrados em sessões semanais de $40 \mathrm{~min}$, por um ano. Os dados das respostas da cuidadora foram submetidos ao Tau-U. Esta estatística revelou que nem todas as respostas da cuidadora, na fase de intervenção, mostraram fortes efeitos do tratamento, enquanto outros atestaram a robustez do mesmo. Com 95\% de probabilidade de acerto, os procedimentos de intervenção afetaram significativamente as seguintes respostas da cuidadora: "usar músicas infantis" e "fazer um comentário engraçado ou elogioso para que a criança voltasse a brincar". Na fase de intervenção, houve um aumento considerável de iniciativas da menina e de respostas da cuidadora, assim como das diversas modalidades de respostas (vocal/verbal, gestual e mista) de ambas. Assim, o programa de formação da cuidadora influenciou de forma significante a frequência de turnos e as modalidades de respostas da criança; e que a intervenção, via cuidadora, orientada por profissional, pode ser efetiva e apropriada ao contexto socioeconômico brasileiro.

PALAVRAS-CHAVE: Transtorno do espectro autista. Intervenção precoce. Abordagem desenvolvimentista.

\section{ABSTRACT}

Autism Spectrum Disorder (ASD) is characterized by persistent deficits in communication and social interaction, besides repetitive patterns of behavior and restricted interests. The purpose of this program was to verify the effect of the application of a parenting teaching program/caregivers about their child's performance. An 18-month-old girl with ASD and her caregiver participated in the program. It is a quasiexperimental single subject research design - $A B$ (baseline, treatment and follow-up). An individualized program was implemented to teach the caregiver how to use playful procedures, aiming to promote social interaction and an increase in the interactive turns between both participants. The behaviors of both

\footnotetext{
${ }^{1}$ Doutora em Educação - Universidade do Estado do Rio de Janeiro (UERJ) - Rio de Janeiro, RJ - Brasil. Pesquisadora - Instituto Federal Fluminense (IFF) - Campos dos Goytacazes, RJ - Brasil. E-mail: odilamansur@yahoo.com.br.

${ }^{2}$ Doutora em Educação Especial - Vanderbilt University - Nashville, Tennessee - Estados Unidos. Docente Universidade do Estado do Rio de Janeiro (UERJ) - Rio de Janeiro, RJ - Brasil. E-mail: leilareginanunes@gmail.com.
}

Submetido em: 27/05/2019 - Aceito em: 10/11/2019

(C) ETD- Educação Temática Digital Campinas, SP $\quad$ v.22 n.1 $\quad$ p.50-67 jan./mar.2020


participants were registered in 40-min weekly sessions for a year. Data from the caregiver's responses were submitted to Tau-U. This statistic revealed that not all the caregiver's responses, in the intervention phase, showed strong effects of the treatment, while others attested its robustness. With $95 \%$ likelihood of success, the intervention procedures significantly affected the following caregiver's responses: "using children's songs" and "making a funny or complimentary commentary to have the child return to play." There was a great increase of child's initiatives and the caregiver's responses as well as both several types of responses (vocal/verbal, gestural and mixed). Thus, the caregiver's training program strongly influenced the turns frequency and the child's types of responses; and that the caregiver intervention, guided by a professional, can be effective and adequate to the Brazilian socioeconomic context.

KEYWORDS Autism spectrum disorder. Early intervention. Developmental approach.

\section{RESUMEN}

El trastorno del espectro del autismo (TEA) se caracteriza por déficits persistentes en la comunicación e interacción social, comportamiento repetitivos e intereses restringidos. El objetivo de este estudio fue verificar el efecto de la aplicación de un programa de enseñanza por padres / cuidadores sobre el desempeño de su hijo. Participaron una niña de 18 meses, con TEA, y su cuidadora. Se trata de una investigación casi experimental, intrasujetos, del tipo AB (línea de base, tratamiento y seguimiento). Se llevó a cabo un programa individualizado para enseñar a la cuidadora a utilizar procedimientos lúdicos, buscando promover la interacción social y el aumento de turnos interactivos de ambos participantes. Los resultados se registraron en sesiones semanales de 40 minutos por un año. Los datos de las respuestas de la cuidadora fueron sometidos al Tau-U. Esta estadística reveló que no todas las respuestas de la cuidadora, en la fase de intervención, mostraron fuertes efectos del tratamiento, mientras que otros atestaron la robustez del mismo. Con un $95 \%$ de probabilidad de acierto, los procedimientos de intervención afectaron significativamente a las siguientes respuestas de la cuidadora: "usar música infantil" y "hacer un comentario divertido o elogioso para que el niño volviera a jugar". En la fase de intervención hubo un aumento considerable de iniciativas de la niña y de respuestas de la cuidadora, así como de las diversas modalidades de respuestas (vocal / verbal, gestual y mixta) de ambas. Así, el programa de formación de la cuidadora influenció de forma significante la frecuencia de turnos y las modalidades de respuestas del niño; y que la intervención, vía cuidadora, orientada por profesional, puede ser efectiva y apropiada al contexto socioeconómico brasileño.

PALAVRAS-CLAVE: Trastorno del espectro del autismo. Intervención precoz. Enfoque desarrollista.

\section{INTRODUÇÃO}

O desenvolvimento infantil pode ser definido como um processo progressivo, multidimensional e integral que se inicia com a concepção e que engloba o crescimento físico, a maturação neurológica, os desenvolvimentos comportamental, sensorial, cognitivo e de linguagem, assim como as relações socioafetivas. Tem como efeito tornar a criança capaz de responder às suas necessidades e as do seu meio, considerando-se seu contexto de vida (OPAS, 2006).

Nem sempre o desenvolvimento infantil ocorre de forma típica. Podem ocorrer atrasos, desvios ou transtornos no desenvolvimento da criança. Dentre estes transtornos encontra-se o autismo. Nos últimos anos, assistimos a alterações consideráveis na classificação diagnóstica do Transtorno do Espectro Autista (TEA), em consequência de 
revisões sistemáticas de critérios, por parte da comunidade científica (APA, 2013), e de avanços clínicos resultantes da prática de profissionais especializados.

A tríade clínica de incapacidades que define o TEA na DSM-IV é reduzida para dois critérios na DSM-5, ficando os déficits de interação social e os da comunicação englobados num único critério, designados como "déficits na comunicação social" além dos "comportamentos e interesses restritos e repetitivos" (APA, 2014).

O TEA tem início precoce e traz dificuldades que tendem a comprometer o desenvolvimento do indivíduo, ao longo de sua vida, apresentando uma extensa variabilidade na intensidade e forma de expressão da sintomatologia, nas áreas que definem o seu diagnóstico. Trata-se de uma síndrome comportamental complexa que apresenta etiologias múltiplas, combinando fatores genéticos e ambientais (RUTTER, 2011).

Desse modo, é preciso que o mais precocemente possível sejam ofertadas intervenções que possam minimizar os prejuízos causados pela ausência de comunicação, de interação social e de simbolização, de forma a melhorar a qualidade de vida da criança e inseri-la em sociedade. Através da intervenção precoce, tem-se como objetivo impedir que os sintomas do autismo tornem-se irreversíveis ou mais difíceis de serem tratados (LEWIS; ELMAN, 2008).

Já que não se pode falar ainda em cura para o autismo, é preciso que se desenvolvam estratégias que deem conta dos prejuízos secundários deste transtorno (HADWIN, BARON-COHEN, 1999). A neuroplasticidade cerebral, que permite maior rearranjo das ligações sinápticas e funcionamento cerebral, é maior nos primeiros anos de vida, então, estimular a criança precocemente pode favorecer a diminuição dos sintomas e aumentar as chances de seu desenvolvimento (MCCONACHIE; WATSON, 2014).

O tratamento engloba inúmeras intervenções educacionais e clínicas, através de uma equipe multidisciplinar, de acordo com as necessidades de cada criança. É possível observar que existe um grande número de possibilidades referentes ao tratamento para o Transtorno do Espectro Autista, podendo este ser medicamentoso ou terapêutico, de base comportamentalista ou desenvolvimentista, ressaltando-se a importância da intervenção precoce de acordo com o perfil de cada criança e de sua família (STUDER et al., 2017).

Assim, a abordagem desenvolvimentista responderia bem a tais dificuldades do autista, porque objetiva que a criança desenvolva habilidades efetivas de comunicação, sejam elas verbais ou não verbais, favorecendo o desenvolvimento da linguagem, em ambientes naturais, por meio da retomada da sequência do desenvolvimento típico inicial. 
Tudo isso ajuda a minimizar problemas comportamentais, como agressões e autolesões, aumentar a interação social, maximizar as condutas intencionais e socioafetivas da criança (GREENSPAN; WIEDER, 1999; MCCONACHIE et al., 2005).

A literatura aponta que o modelo desenvolvimentista tem-se revelado eficaz em favorecer o desenvolvimento das crianças, especialmente entre 18 e 48 meses, e pode ser utilizado em contextos naturais. Ele fornece inúmeras estratégias estruturadas para trabalhar com crianças muito jovens, em contextos individuais e em grupo, promovendo o seu desenvolvimento em domínios-chave como a imitação, a comunicação, a cognição, as competências motoras e sociais, o comportamento adaptativo e os jogos (LAMPREIA, 2008; WETHERBY; PRIZANT, 2000), daí a nossa opção por utilizá-la nesta pesquisa.

Assim, ao longo das décadas, o atendimento às crianças autistas passou a ter como foco o início do transtorno, antes que os prejuízos sejam agravados e tornem o tratamento mais difícil. Desta forma, as intervenções atuais, desenvolvidas em outros países, como Estados Unidos e Inglaterra, utilizam a abordagem desenvolvimentista, atuando tão logo as dificuldades iniciais são apontadas (DAWSON; ZANOLLI, 2003).

De forma a consolidar estes conhecimentos sobre o autismo, realizamos esta pesquisa. Mais especificamente, este estudo pretendeu avaliar os efeitos da aplicação de um programa de intervenção precoce, para pais/ cuidadores, baseado na abordagem desenvolvimentista, na ocorrência de turnos e de modalidade de iniciativas e respostas para crianças com menos de trinta meses de idade, com diagnóstico ou suspeita de autismo.

\section{MÉTODO}

\subsection{Participante: Izabel}

A pesquisa contou com três crianças e respectivos cuidadores. Neste artigo, no entanto, será descrito o trabalho somente com Izabel, por ser, dentre as três crianças, a que apresentava um quadro clínico mais grave, bem como a mais jovem a ser inserida nas intervenções. A participante era criança atendida pelos pediatras, no ambulatório de Seguimento em Pediatria, da Faculdade de Medicina de Campos, aos 18 meses, pelo neurologista e pelo pediatra, com diagnóstico de autismo, apresentando quadro severo. Além da menina, com sua respectiva cuidadora participaram também deste estudo uma Terapeuta Ocupacional e uma Pedagoga, que atuavam no referido Ambulatório.

\subsection{Local da pesquisa}


Esta pesquisa se desenvolveu no supracitado ambulatório, situado em Campos dos Goytacazes-RJ. Este Serviço da rede SUS (Sistema Único de Saúde) recebe crianças de 1 a 7 anos para tratamento do Transtorno do Espectro. Neste serviço há uma equipe multidisciplinar, composta por pedagogos, psicopedagogos, fisioterapeuta, psicólogo e terapeuta ocupacional. $\mathrm{O}$ ambulatório possui três salas para as intervenções.

\subsection{Materiais e instrumentos}

Para a implementação do protocolo foram utilizados uma filmadora digital Sony (Manducam); jogos pedagógicos, brinquedos variados (sonoros, musicais, jogos de encaixe e outros); fantasias; óculos; peruca colorida; "tapete mágico"; material para exploração sensorial e fichas para registro cotidiano das sessões, bem como "diário de campo".

Os instrumentos de rastreio aplicados foram o M-CHAT e o ESAT.

\subsection{Procedimentos específicos}

Após aprovação pelo Comitê de Ética em Pesquisa (protocolo 35454314.4.0000.5282), foi iniciada a pesquisa.

O estudo foi desenvolvido em três fases: linha de base, tratamento e follow-up.

a) Fase de linha de base

Ao adentrarem o Serviço, a mãe foi entrevistada sobre: gravidez, parto, doenças pré e pós-natais, doenças que a criança já teve; frequência à Creche/Escola; local de residência; pessoas com quem a criança mora; motivo do encaminhamento ao Serviço; percepção da mãe sobre o que é "diferente" em seu filho; uso de medicamentos; descrição de como a criança se alimenta, brinca, dorme, contato lúdico com outras crianças, fala, indicando a idade em que iniciou a fala, dentre outros.

A seguir, a criança foi estimulada pelo terapeuta a brincar: este propunha brincadeiras e jogos, de forma a conhecê-la. Também foram aplicados testes de rastreio: $M$ Chat e ESAT; bem como observação sobre o perfil sensorial da criança, com ajuda da família. Depois, a criança foi encaminhada às terapias com a terapeuta ocupacional, a psicopedagoga e a cuidadora.

Foram então conduzidas 10 sessões com duração de 40 minutos da cuidadora e a criança, sem nenhuma orientação prévia da pesquisadora. A cuidadora foi solicitada a brincar com a criança utilizando ou não os brinquedos e materiais existentes na sala do Ambulatório Interdisciplinar. Esta fase foi encerrada após indicação de certa habilidade da cuidadora na organização do ambiente, de forma a favorecer a comunicação da criança, por

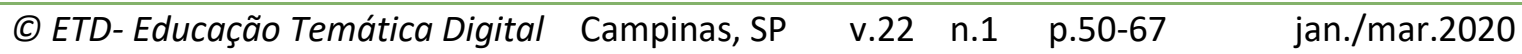


meio da disposição dos brinquedos/objetos dentro do campo visual da criança e fora do seu alcance.

b) Fase de intervenção

Nesta fase, a cuidadora e as terapeutas foram chamadas a participar de um Curso de Capacitação com duração de oito horas e de um programa de intervenção aplicado aos pais e cuidadores.

\section{Etapa 1 - Curso de Capacitação I, para pais/cuidadores}

O objetivo desse curso foi orientar pais a promover o desenvolvimento das crianças participantes, por meio da oferta de novas situações de aprendizagem, de modo a se tornarem coterapeutas, reproduzindo as orientações recebidas e possibilitando a emissão dos comportamentos adequados em outros contextos.

\section{Etapa 2 - Programa de intervenção aplicado à cuidadora}

Foram realizadas 15 sessões interventivas com a criança, sua cuidadora a e as terapeutas. A fase de intervenção sobre a criança compreendeu dois procedimentos e teve como objetivo ajudar a criança a emitir espontaneamente respostas pré-verbais, bem como melhorar sua interação social com os cuidadores e terapeutas.

Os objetivos dessa intervenção foram: a) identificar áreas e repertório de comportamentos das crianças a serem desenvolvidos ou comportamentos a serem extintos por meio da análise das sessões videografadas durante a linha de base e de vídeos caseiros trazidos pelos pais; b)identificar áreas e repertório de comportamentos dos adultos, no tratamento com a criança com autismo, que devem ter continuidade ou que precisam ser modificados; c) possibilitar a troca de informações entre os pais, de forma a auxiliar a dirimir dúvidas de forma a auxiliar no desenvolvimento da criança com autismo; d) elaborar um programa individualizado, estimulando o aprendizado de novas habilidades, ampliando os repertórios de potencialidades e reduzindo comportamentos mal adaptativos e disfuncionais, bem como favorecendo interações sociais mais positivas e melhorando a qualidade do padrão de comunicação.

Utilizando os vídeos, a cuidadora, a pesquisadora e as terapeutas puderam identificar os principais aspectos a serem observados para implementação das estratégias de ensino e registro das respostas da criança, compreendendo o funcionamento de cada uma. Incluímos, no referido programa, os elementos críticos para uma melhor prática nos Programas de Intervenção Precoce destinados a crianças até cinco anos de idade com risco

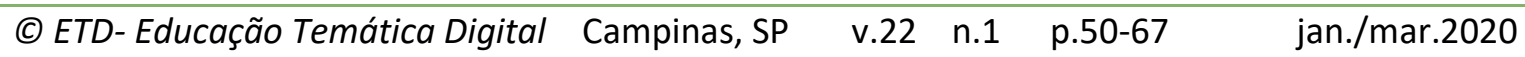


de atraso ou desvio em seu desenvolvimento (LAMPREIA, 2007; NRC, 2001), baseado no perfil individualizado de cada criança (NRC, 2001); centrados no desenvolvimento da atenção (focalizada e sustentada; compartilhada e conjunta); utilização da imitação e uso da linguagem expressiva; emprego de atividades lúdicas/jogo com brinquedos apropriados para favorecer a interação social (NRC, 2001); oferecendo apoios aos pais/cuidadores (GURALNICK, 1998), envolvendo os mesmos como agentes ativos na intervenção (ARAÚJO, 2012; CORREA NETO, 2017; GREENSPAN; WIEDER, 1999; GURALNICK, 1998; MCCONACHIE et al., 2005; NRC, 2001).

Nas 15 sessões de intervenção para cada uma das três crianças foram utilizados três procedimentos:

1. Na primeira parte da sessão, com duração total de 20 minutos, em média, foram estruturadas situações pelas terapeutas com a cuidadora, de modo a propiciar o desenvolvimento das categorias de comunicação inicial das crianças, por meio da intervenção direta da cuidadora a sobre ela;

2. Assim, os 20 minutos restantes eram dedicados à intervenção direta da terapeuta sobre a criança, com a mãe apenas observando suas intervenções, para que ela servisse de modelo de como a mãe poderia agir com a criança em seu dia a dia;

3. Terminados os 40 minutos de intervenção, durante mais 10 minutos, a cuidadora era convidada a refletir, com a pesquisadora e a terapeuta, de que forma poderia corrigir eventuais erros cometidos por ela. A cuidadora era orientada sobre como proceder nas determinadas situações e incentivada a promover as interações com a sua criança, em casa ou nos demais ambientes de convivência.

Fase de follow-up

Esta fase ocorreu 40 dias após o término da intervenção de forma a verificar a manutenção das respostas dos participantes, sem a presença da pesquisadora. Após, foi realizada uma entrevista, filmada e transcrita, com a cuidadora e terapeutas, de forma a expor e avaliar os avanços de cada criança.

\subsection{Delineamento da pesquisa}

Segundo Nunes Sobrinho e Naujorks (2001); Nunes e Walter (2014), trata-se de pesquisa quase experimental, intrassujeitos, do tipo $A B$ - linha de base, tratamento e followup-. Neste tipo de pesquisa, o desempenho de cada sujeito é avaliado em relação a ele mesmo, e não em comparação com os demais. Um único participante é avaliado diversas (C) ETD- Educação Temática Digital Campinas, SP $\quad$ v.22 n.1 $\quad$ p.50-67 jan./mar.2020 
vezes no decorrer do estudo, em vários aspectos diferentes e o nível de desempenho do participante é comparado com ele próprio sob diversas condições experimentais. Estudos com esse tipo de delineamento podem ocorrer em três fases: linha de base (pré-teste), tratamento (intervenção) e follow up (pós-teste). A análise e interpretação dos dados ocorreram de forma quantitativa e qualitativa.

As variáveis desse estudo foram:

$\checkmark$ Variável independente: oferta de programa de capacitação para pais/cuidadores.

$\checkmark$ Variáveis dependentes: desempenho dos pais/cuidadores na interação com as crianças, e que pode ser avaliado em termos da frequência dos seguintes comportamentos:

- usar acessórios divertidos para chamar a atenção da criança: óculos, perucas...;

- usar brinquedos sonoros, coloridos, que emitem som ou luz, ou que são musicais;

- chamar a criança pelo nome diversas vezes;

- ficar em frente do espelho com a criança, ajudar a reconhecer-se pelo nome;

- brincar com a criança, tocá-la, fazer cócegas, de forma a estimular o sorriso responsivo; deixar a criança começar uma brincadeira;

- fazer um comentário engraçado ou elogioso para que a criança o olhe e volte a brincar;

- fazer imitações das ações da criança, repetir sons, vocalizar sílabas;

- usar músicas infantis; a partir de músicas, estimular a imitação dos gestos.

Num segundo momento, o uso de estratégias aprendidas durante a capacitação, pelos pais/cuidadores funcionou como variável independente e a resposta das crianças como variável dependente:

$\checkmark$ Variável independente: desempenho dos pais e terapeutas.

$\checkmark$ Variável dependente (comportamento-alvo): iniciativas de interação e respostas das crianças.

\subsection{Registro e análise dos dados}

Para o registro de todos os dados, foram utilizadas três fichas:

a) Uma de registro de evento, realizado a partir da transcrição das sessões videografadas, o qual envolvia anotar em cada sessão a ocorrência de cada comportamento ao longo dos minutos 06 a 21, tanto da linha de base, quanto da 
intervenção e do follow-up, já que os cinco minutos iniciais eram de ambientação da criança. Os seguintes comportamentos: foram registrados: iniciativa do adulto, modalidade da iniciativa (verbal, gestual, mista (verbal+gestual) do adulto ou da criança, modalidade da resposta (verbal, gestual, mista (verbal+gestual) do adulto ou da criança, bem como as seguintes categorias: imitação; respeito à alternância de turno;

b) uma segunda ficha para fazer o cômputo geral ou síntese das interações e respostas da criança;

c) uma terceira ficha para registrar se os itens do Programa de Intervenção Aplicado aos Pais e Cuidadora foram utilizados na linha de base, na intervenção e no follow-up.

Usou-se um "diário de campo" para anotação de aspectos relevantes das sessões ou situações relatadas pela cuidadora e profissionais.

Os dados das respostas das cuidadoras e das crianças foram submetidos ao Tau-U que é um método para avaliar o tamanho do efeito de uma intervenção. Ele representa um novo índice para análise de dados de pesquisa intrassujeitos que combina a não sobreposição entre fases com a tendência dos dados da fase de intervenção. O Tau-U mensurando dados não sobrepostos entre duas fases (linha de base e intervenção) constitui técnica não paramétrica "livre de distribuição", com poder estatístico de 91\% a 95\% de regressão linear (OLS) quando os dados estão em conformidade com as suposições paramétricas. Este é um índice bem adequado para conjuntos de dados pequenos (PARKER; VANNEST; DAVIS; SAUBER, 2011).

\section{RESULTADOS}

Perfil de Izabel

Izabel, de 18 meses, quando iniciou o tratamento, permaneceu por vários dias sem entrar na sala de atendimentos, mesmo com a mãe. A menina se recusava e chorava muito. Jamais sorriu nesta fase. Não atendia pelo nome. Apresentavas estereotipias. Não fixava o olhar no interlocutor. A tentativa de intervenção (acolhimento) era realizada no corredor do hospital. Izabel estabeleceu grande empatia com a terapeuta ocupacional. Foram realizadas três reuniões com a mãe, a terapeuta ocupacional, a pedagoga e o pediatra da menina, de forma a conhecermos melhor seu quadro clínico. 
Seu repertório de interesses era bastante limitado. Izabel não falava e se comunicava muito pouco. Nos primeiros dias, apesar dos vários brinquedos e brincadeiras propostas, Izabel permaneceu toda a sessão de 30 minutos no colo da mãe, parada, sem se interessar por nenhum dos brinquedos oferecidos (pianinho colorido; Peppa Pig; telefone, livros infantis, jogos de encaixe...) e chorando. A mãe demonstrou desconforto e inquietação, mas, ao mesmo tempo, dizia que queria muito ajudar a filha a melhorar.

$\mathrm{Na}$ linha de base foram realizadas 10 sessões de aproximadamente quarenta minutos cada. Depois, foram realizadas 15 sessões de intervenção, com a participação da cuidadora a e terapeuta e cinco sessões de follow-up. Desta forma, a partir dos instrumentos de avaliação aplicados (M CHAT, ESAT), e fundamentação nas abordagens naturalística e desenvolvimentista, foram elaborados os objetivos e estratégias de ensino para Izabel, visando aumentar atenção ao estímulo social e às funções comunicativas da menina.

Na Figura 1 está apresentado o número de ocorrências das iniciativas de Izabel e das respostas da cuidadora a estas iniciativas, em cada sessão, nas fases de linha de base, intervenção e follow-up.

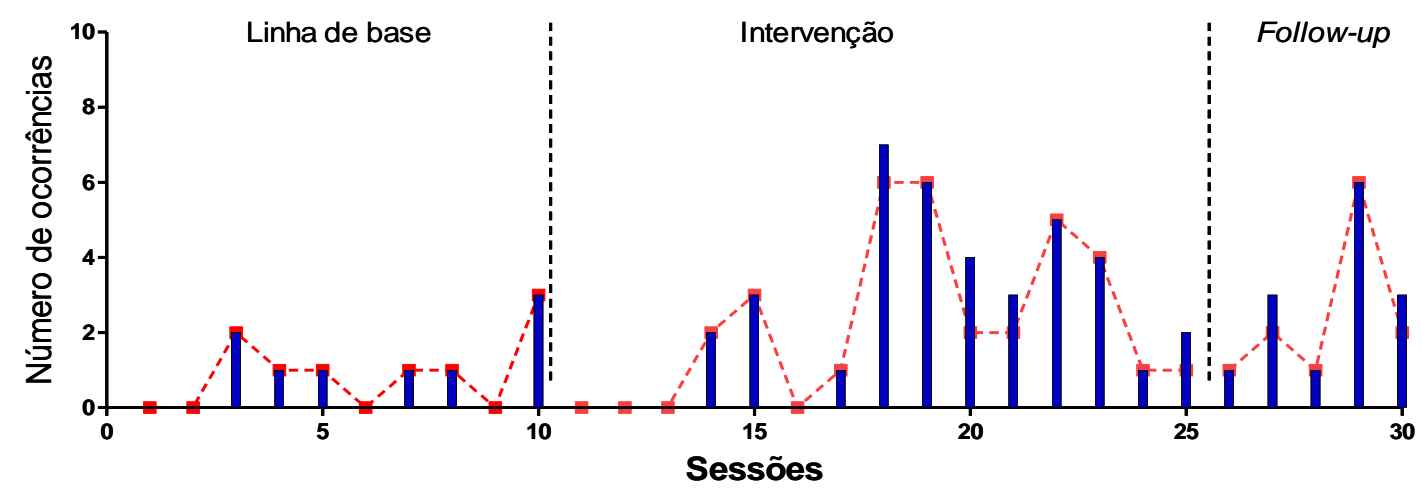

Figura 1 - Número de ocorrências das iniciativas de Izabel e das respostas da cuidadora a estas iniciativas, em cada sessão, nas fases de linha de base, intervenção e follow-up Fonte: os autores, pesquisa de campo

Podemos observar que, na fase da intervenção, assim como no follow up, Izabel aumentou consideravelmente suas iniciativas de interação com a cuidadora, comparativamente à linha de base. A cuidadora a respondeu a estas iniciativas nas três fases. 
A tabela 1 apresenta o somatório das modalidades de respostas verbal/vocal; gestual (incluindo expressão facial, expressão corporal) e misto (vocal + gestual) empregadas pela díade durante todas as sessões de linha de base, intervenção e follow-up.

Tabela 1 - Frequência total das modalidades de respostas emitidas pela díade nas diversas atividades

\begin{tabular}{lrrrrrr}
\hline & \multicolumn{3}{c}{ Cuidadora } & & \multicolumn{3}{c}{ Izabel } \\
\hline & $\begin{array}{c}\text { Linha de } \\
\text { Base }\end{array}$ & Intervenção & Follow-up & $\begin{array}{c}\text { Linha de } \\
\text { Base }\end{array}$ & Intervenção & Follow-up \\
\hline Verbal/Vocal & 16 & 46 & 14 & 17 & 21 & 4 \\
Exp. Facial & 12 & 9 & 1 & 34 & 77 & 23 \\
Exp. Corporal & 39 & 72 & 20 & 30 & 87 & 32 \\
Mista & 41 & 90 & 32 & 27 & 37 & 7 \\
Total & 108 & 217 & 67 & 108 & 222 & 66 \\
\hline
\end{tabular}

Fonte: os autores, pesquisa de campo

Houve um aumento generalizado nas diversas modalidades de respostas, tanto da cuidadora quanto da criança, na fase de intervenção comparativamente à de linha de base. No follow-up, contudo, houve um decréscimo em todas as modalidades.

$\mathrm{Na}$ Tabela 2 são apresentados os dados do Tau-U acerca dos comportamentos/estratégias utilizados pela cuidadora de Izabel, nas sessões de intervenção, comparativamente à fase de linha de base.

Tabela 2 - Dados do Tau-U acerca do aumento estatisticamente significante do emprego de cada estratégia de ensino nas sessões de intervenção

\begin{tabular}{|c|c|c|}
\hline Estratégias de ensino & $p$ & TAU-U \\
\hline Usa acessórios divertidos para buscar chamar a atenção da criança & 0,1655 & $-0,3333$ \\
\hline Usa brinquedos sonoros & 0,6774 & 0,1000 \\
\hline Chama a criança pelo nome & 0,2120 & 0,3000 \\
\hline $\begin{array}{l}\text { Fica em frente ao espelho com a criança, ajuda a reconhecer-se } \\
\text { pelo nome }\end{array}$ & 0,4881 & 0,1667 \\
\hline Brinca com a criança pelo toque, estimulando o sorriso responsivo & 0,6774 & 0,1000 \\
\hline
\end{tabular}


Deixa a criança começar uma brincadeira

0,0522

0,4667

Faz um comentário engraçado ou elogioso para que a criança volte

$0,0036^{*}$

0,7000 a brincar

Faz imitações, repete sons, parecidas com as da criança

$0,4054 \quad 0,2000$

Estimula a imitação, alternando turnos

$0,1655 \quad 0,3333$

Usa músicas infantis

0,6774

0,1000

A partir das músicas, estimula a imitação dos gestos

0,8897

0,0333

* Diferença estatisticamente significante, corte em $\mathrm{p}<0,05$

Fonte: os autores, pesquisa de campo

O teste estatístico mostra um forte efeito do Curso de Capacitação para Cuidadora as no comportamento de fazer comentários engraçados ou elogiosos para que a criança voltasse a brincar e um efeito relativamente fraco quanto ao comportamento de deixar a criança começar uma brincadeira. Não houve, todavia, diferença estatisticamente significante quanto às demais variáveis.

Na Tabela 3 estão resultados do Tau-U sobre a ocorrência de diferentes modalidades de iniciativa e de resposta de Izabel na fase de intervenção comparativamente à de linha de base

Tabela 3 - Resultados do Tau-U acerca do aumento estatisticamente significante da ocorrência de diferentes modalidades de iniciativa e de resposta de Izabel na fase de intervenção comparativamente à de linha de base (corte em $p<0,05$ )

\begin{tabular}{llc}
\hline Izabel & \multicolumn{2}{l}{} \\
\hline Iniciativa & $p$ & TAU-U \\
Verbal & 0,5791 & $-0,1333$ \\
Facial & $\mathbf{0 , 0 2 6 5}$ & $\mathbf{0 , 5 3 3 3}$ \\
Corporal & 0,1077 & 0,3867 \\
Mista & 0,4881 & 0,1667 \\
Total & 0,0855 & 0,4133 \\
Resposta & $p$ & TAU-U \\
Verbal & 0,6572 & $-0,1067$ \\
Facial & $\mathbf{0 , 0 3 0 5}$ & $\mathbf{0 , 5 2}$ \\
Corporal & $\mathbf{0 , 0 1 9 8}$ & $\mathbf{0 , 5 6}$ \\
Mista & 0,4054 & $-0,2$ \\
Total & 0.0714 & 0,4333 \\
\hline
\end{tabular}

Fonte: os autores, pesquisa de campo 
O teste estatístico mostrou efeitos relativamente moderados do Curso de Capacitação para Cuidadora nas iniciativas, usando expressões faciais, e nas respostas faciais e corporais de Izabel. Não houve, todavia, diferença estatisticamente significante quanto às demais variáveis.

Os comportamentos de "prestar a atenção aos estímulos sociais, "alternância de turno e comportamento antecipatório" parecem ser de máxima importância para que a criança realmente se engaje em trocas sociais recíprocas que possibilitarão o seu desenvolvimento e, conforme observado durante a aplicação do programa, estes comportamentos parecem estar relacionados com o papel da criança em se tornar o iniciador das interações, que é um dos princípios fundamentais para o desenvolvimento infantil, conforme preconizam Wetherby, Schuler e Prizant (2000).

Foram evidenciados ganhos nas iniciativas da menina, por meio das expressões faciais. Especialmente, em jogos corporais, como "Serra, serra, serrador", a menina esboçava sorriso farto. Por meio da expressão facial a menina solicitava que o jogo não parasse, por exemplo. Também, na hora de ir embora, passou a olhar para a cuidadora, dar a mão a esta e se encaminhar para a porta de saída. Estes devem ser os primeiros comportamentos observados como alvo das estratégias da intervenção.

Para avaliar a generalização dos comportamentos aprendidos pela cuidadora e pela criança é imprescindível que o profissional avalie a díade em sessões mais formais em outros contextos. Entretanto, não foi possível levar a criança para outro ambiente.

$\mathrm{Na}$ fase de follow up foram oferecidas atividades um pouco diferentes das realizadas anteriormente e mostrados brinquedos novos pela cuidadora. Contudo, a menina estava retornando de uma internação hospitalar, por problemas pulmonares, um tanto debilitada, e isto se refletiu em resultados menos exuberantes nesta fase de follow up do que na fase de intervenção.

\section{VALIDADE SOCIAL}

A validade social refere-se à percepção dos próprios participantes, de seus pais e profissionais acerca das mudanças na vida dos participantes em decorrência da intervenção. Ela implica igualmente na manutenção dos ganhos após a finalização da pesquisa, ou seja, se a intervenção apresenta "sustentabilidade de efeitos" (NUNES, 2010).

Foi realizada uma entrevista com os pais da criança envolvida, após as intervenções, com a participação das terapeutas e da pesquisadora. Estas entrevistas foram filmadas e transcritas. De modo sintético, na visão dos pais, identificamos validade social deste estudo, que pode ser identificada na fala abaixo, de forma tímida, mas animadora para futuras pesquisas, quando a mãe relata: “... depois do tratamento aqui, ela agora já é mais sociável,

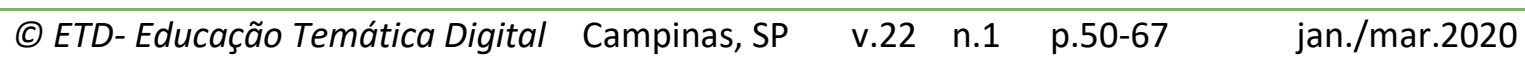


fica até feliz de sair. Ela gosta de ir para a rua, tem que ver, pega a minha mãozinha... $\mathrm{Na}$ escola, antes, ficava atrás da porta da sala se escondendo das outras crianças..."

A validade social deste estudo está, além dos ganhos para o desenvolvimento da criança, em relação ao procedimento com a cuidadora. Esta se tornou parceira ativa das estratégias da intervenção, pois a manutenção e a generalização dos comportamentos apresentados durante as sessões formais são a garantia de que a criança os desenvolveu e os utilizará em outros contextos (GURALNICK, 2000).

\section{DISCUSSÃO E CONCLUSÃO}

São vários os estudos que indicam a intervenção precoce como fator primordial para a melhora do quadro clínico do autismo, gerando ganhos significativos e duradouros no desenvolvimento da criança (CHARMAN, 2004; REICHOW, 2011). Devido à neuroplasticidade cerebral, a intervenção iniciada precocemente desempenha papel importante, fortalecendo os efeitos positivos da mesma. Dentre os ganhos decorrentes da intervenção precoce, quando estudada a longo prazo, estão redução dos gastos dos familiares no tratamento das crianças, redução de gastos para a saúde pública, melhores resultados para a criança e minimização do quadro autístico, segundo Garcia e Lampréia (2011).

É, então, de grande importância a implementação de medidas preventivas e intervenção precoce com bebês de risco. É necessária uma escuta qualificada da família e da pessoa em questão, incluindo: sua história de vida (dados sobre a gestação, o nascimento, os primeiros anos de vida, os marcos de desenvolvimento); a configuração familiar (quem mora na casa, laços familiares, relações com amigos, quem se ocupa prioritariamente do cuidado); sua rotina diária (Creche, Escola ou grupo social, dia a dia, autonomia); sua história clínica, intercorrências de saúde, hospitalizações, etc., segundo o Ministério da Saúde (BRASIL, 2015).

Porém, vários fatores podem retardar a intervenção, como a demora na detecção das primeiras dificuldades no comportamento da criança, na busca pela ajuda profissional e na realização do diagnóstico, o que limita uma quantidade maior de pesquisas referentes à intervenção precoce no TEA. Alguns estudos têm demonstrado que crianças com autismo poucas vezes recebem esse diagnóstico antes dos cinco anos (DALEY, 2004; MANDELL; LISTERUD; LEVY; PINTO-MARTIN, 2002), sendo que algumas são diagnosticadas apenas quando atingem idade escolar (NOTERDAEME; HUTZELMEYER-NICKELS, 2010).

A intervenção, via cuidadores, orientada por profissional, pode ser efetiva e apropriada ao contexto socioeconômico brasileiro, que carece de programas governamentais e de profissionais qualificados para conduzir intervenções no tratamento de autismo precoce (AUTOR, 2018). 
Porém, como limitante ao êxito deste estudo, encontramos dificuldades em fomentar momentos com o cuidador, individualmente, acreditando ser necessária uma atenção específica para a família, na ausência da criança, de modo que ela tenha um espaço exclusivo para trabalhar as suas próprias questões.

Dentre os fatores que limitaram, de certo modo, o desenvolvimento da pesquisa, destacamos: dificuldades em fomentar momentos particulares com o cuidador sem a presença da criança, com o propósito de oferecer-lhe um espaço exclusivo para trabalhar as suas próprias questões; impedimentos das famílias em participar das sessões de pesquisa mais frequentemente e por um período de tempo mais extenso; pouca disponibilidade das famílias em receber a pesquisadora em seus lares.

O presente trabalho aponta uma contribuição relevante para o desenvolvimento de novos programas de intervenção precoce que poderão ajudar outras crianças que apresentam transtornos em seu desenvolvimento, sabendo-se que muito ainda precisa ser investigado e aperfeiçoado.

Ressalta-se, ainda, a necessidade de um maior investimento na realização de estudos experimentais e quase experimentais em educação e em saúde. Sugere-se que novos estudos sejam realizados, reforçando-se a necessidade de pesquisas rigorosas, que apontem os reais benefícios da intervenção precoce no autismo. O diagnóstico precoce do TEA é um enorme desafio no Brasil, o que dificulta a realização de pesquisas com crianças em tenra idade.

\section{REFERÊNCIAS}

AMERICAN PSYCHIATRIC ASSOCIATION. APA. Transtornos mentais: DSM-V. In: AMERICAN PSYCHIATRIC ASSOCIATION. Manual diagnóstico e estatísticos de transtornos mentais.

5. ed. Porto Alegre: Artmed, 2013.

ARAUJO, Álvaro; LOTUFO NETO, Francisco. A nova classificação americana para os transtornos mentais: o DSM. Revista Brasileira de Terapia Comportamental e Cognitiva, São Paulo, v. 16, n. 1, abr. 2014.

BARTON, Marianne L. et al. Sensitivity and Specificity of Proposed DSM-5 Criteria for Autism Spectrum Disorder in Toddlers. Journal of Autism and Developemental Disorders, New York, v.43, n.5, p.1184-1195, 2013.

BRASIL. Ministério da Saúde. Linha de cuidado para a atenção às pessoas com transtornos do espectro do Autismo e suas famílias na rede de atenção psicossocial do sistema único de saúde. Brasília, DF: Ministério da Saúde, 2015.

CHARMAN, Tony. Why is joint attention a pivotal skill in autism? In: FRITH, U.; HILL, E.L. Autism: mind and brain. Oxford: Oxford University, 2004. 
CORRÊA NETO, Márcia Miriam. Práticas interventivas precoces de comunicação para crianças com Transtorno do Espectro Autista (TEA): um estudo quase experimental. 2017. Tese (Doutorado em Educação) - Faculdade de Educação, Universidade do Estado do Rio de Janeiro, Rio de Janeiro, 2017.

DALEY, Tamara. From symptom recognition to diagnosis: children with autism in urban India. Social Science \& Medicine, v.58, p.1323-1335, 2004.

DAWSON, Geraldine; ZANOLLI, Kthleen. Early intervention and brain plasticity in Autism. In: BOCK, Gregory R.; GOODIE, Jamie A. (Ed.). Autism: neural basis and treatment possibilities. New York: John Wiley \& Sons, 2003.

GARCIA, Mariana Luiza; LAMPREIA, Carolina. Limites e possibilidades da identificação de risco de Autismo no primeiro ano de vida. Psicologia Reflexão e Crítica, Porto Alegre, v. 24, n. 2, p. 300-308, 2011. DOI: http://dx.doi.org/10.1590/S0102-79722011000200011. Disponível em: http://www.scielo.br/scielo.php?script=sci arttext\&pid=S010279722011000200011\&lng=pt\&nrm=iso . Acesso em: 13 mar. 2016.

GREENSPAN, Stanley I.; WIEDER, Serena. Developmental patterns and outcomes in infants and children with disorders in relating and communicating: a chart review of 200 cases of children with autistic spectrum diagnoses. Journal of Developmental and Learning Disorders, v.1, p. 87-141, 1999.

GURALNICK, Michel. J. Early childhood intervention: evolution of a system. Focus on autism and other Developmental Disabilities, v. 15, n.2, p. 68-79, 2000.

HEDLEY, Darren et al. Cross-cultural evaluation of the Autism Detection in Early Childhood (ADEC). Autism, v. 14, n.2, p. 93-112, 2010.

HILL, Elisabeth L.; FRITH, Uta. Understanding autism: insights from mind and brain.

Philosophical Transactions of The Royal Society of London. Series B. Biological Sciences, v. 358, p. 281-289, 2003.

HOWLIN, Patricia; HADWIN, Julie; BARON-COHEN, Simon. Teaching children with autism to mind-read: a practical guide for teachers and parents. New York: John Wiley \& Sons, 1999.

LAMPREIA, Carolina. O processo de desenvolvimento rumo ao símbolo: uma perspectiva pragmática. Arquivo Brasileiro de Psicologia, Rio de Janeiro, v. 60, n. 2, p. 117-128, jun. 2008. Disponível em: http://pepsic.bvsalud.org/scielo.php?script=sci arttext\&pid=S1809$\underline{52672008000200012 \& \operatorname{lng}=p t \& n r m=i s o}$. Acesso em: 29 maio 2016.

LAMPREIA, Carolina. A perspectiva desenvolvimentista para a intervenção precoce no Autismo. Estudos de Psicologia, Campinas, v. 24, n.1, p.105-114, mar. 2007. ISSN 0103166X. DOI: http://dx.doi.org/10.1590/S0103-166X2007000100012. Disponível em http://www.scielo.br/scielo.php?script=sci arttext\&pid=S0103166X2007000100012\&lng=pt\&nrm=iso . Acesso em: 18 maio 2016. 
LEWIS, John D.; ELMAN, Jeffrey L. Growth-related neural reorganization and the autism phenotype: a test of the hypothesis that altered brain growth leads to altered connectivity. Developmental Science, v. 11, p. 135-155, 2008. DOI: 10.1111/j.1467-7687.2007.00634.x.

LORD, Catherine; McGEE, James (Ed.). Educating children with autism. Washington, DC: National Academy Press, 2001.

MANDELL, David S. et al. Race differences in the age among medicaid-eligible children with autism. Journal of American Academy of Child and Adolescent Psychiatry, v.41, p.14471453, 2002.

MANSUR, Odila Maria Ferreira Carvalho. Falando com bebês: da detecção de riscos para autismo à intervenção precoce.2018. Tese (Doutorado) - Universidade do Estado do Rio de Janeiro, RJ, 2018.

MATSON, Jonnhy L. et al. A comparison of social and adaptive functioning in persons with psychosis, autism, and severe or profound mental retardation. Journal of Developmental and Physical Disabilities, v. 15, n. 1, p. 57-65, 2003.

MATSON, Johnny L.; DEMPSEY, Timothy; LOVULLO, Santino. V. Characteristics of social skills for adults with intellectual disability, autism and PDD-NOS. Research in Autism Spectrum Disorders, v. 3, n. 1, p. 207-213, 2009.

MCCONACHIE, Helen; FLETCHER-WATSON, Sue. Building capacity for rigorous controlled trials in autism: the importance of measuring treatment adherence. Child: Care, Health and Development, v. 41, v.2, p.169-177, 2014. DOI: 10.1111/cch.12185.

NATIONAL AUTISM CENTER (Ed.). Evidence-based practice autism in the schools: a guide to providing appropriate interventions to students with autism spectrum disorders. 2. ed. Randolph, Massachusetts: National Autism Center, 2011.

NOTERDAEME, Michele; HUTZELMEYER-NICKELS, Anna. Early symptoms and recognition of pervasive developmental disorders in Germany. Autism, v.14, n.6, p. 575-588, 2010.

NUNES SOBRINHO, Francisco de Paula; NAUJORKS, Maria Inês. (Org.). Pesquisa em educação especial: o desafio para a qualificação. v.1, Bauru, SP: EDUSC, 2001.

NUNES, Débora Regina de Paula; ARAÚJO, Eliana Rodrigues. Autismo: a educação infantil como cenário de intervenção. Dossiê Educação Especial: diferenças, currículo e processos de ensino e aprendizagem. Arquivos analíticos de políticas educativas, v.22, n. 84, ago. 2014

NUNES, Débora Regina de Paula; ARAÚJO, Eliana Rodrigues. Autismo: a educação infantil como cenário de intervenção. Education Policy Analysis Archives/Archivos Analíticos de Políticas Educativas, v. 22, p. 1-14, 2014.

NUNES, Débora Regina de Paula; NUNES SOBRINHO, Francisco de Paula. Comunicação alternativa e ampliada para educandos com Autismo: considerações metodológicas. Revista Brasileira de Educação Especial, Marília, v. 16, n. 2, p. 297-312, maio/ago. 2010.

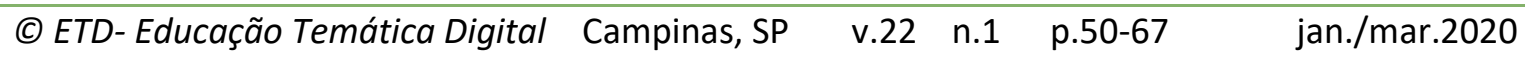


OPAS. Atenção integrada às doenças prevalentes da infância. In: OPAS. Manual para vigilância do desenvolvimento infantil no contexto da AIDPI. Washington, D. C.: OPAS, 2006.

PARKER, Richard I. et al. Combining nonoverlap and trend for single-case research: Tau-U. Behavior Therapy, v. 42, n. 2, p. 284-299, 2011.

PEREIRA, Alessandra et al. Autismo infantil. Tradução e validação da Childhood Autism Rating Scale para uso no Brasil. Jornal de Pediatria, Rio Janeiro, [online], v. 84, n. 6, p. 487494, 2008. ISSN 0021-7557. DOI: http://dx.doi.org/10.1590/S0021-75572008000700004.

PEREIRA, Manuela. Autismo: uma perturbação pervasiva do desenvolvimento. Lisboa: Gaia, 2008. (Colecção Biblioteca do Professor, Gailivro, v.n.).

REICHOW, Brian. Overview of meta-analyses on early intensive behavioral intervention for young children with autism spectrum disorders. Journal of Autism and Development Disorders, v. 42, n. 4, p. 512-520, 2011.

ROGERS, Sally J.; BENNETTO, Loisa. Intersubjectivity in autism: the roles of imitation and executive function. WETHERBY, A. M.; PRIZANT, B. M. (Org.). Autism spectrum disorders: a transactional developmental perspective. Baltimore: Paul H. Brookes, 2000.

RUTTER, Michael L. Progress in understanding autism: 2007-2010. Journal of Autism and Developmental Disorders, v. 41, p. 395-404, 2011

STUDER, Nadja, et al. Implementation of early intensive behavioural intervention for children with autism in Switzerland. BMC Psychiatry, v. 17, n. 1, 2017.

WALTER, Cátia Criveland; NUNES, Leila Regina de Paula. Pesquisa experimental em educação especial. In: Nova Trilhas no modo de fazer pesquisa em Educação Especial. São Carlos, SP: Marquezine \& Manzini, ABPEE, 2014.

ZANON, Regina Basso; BACKES, Bárbara; BOSA, Cleonice Alves. Identificação dos primeiros sintomas do Autismo pelos pais. Psicologia: Teoria e Pesquisa, Brasília, v. 30, n. 1, p. 25-33, mar. 2014. DOI: http://dx.doi.org/10.1590/S0102-37722014000100004. Available from http://www.scielo.br/scielo.php?script=sci arttext\&pid=S010237722014000100004\&lng=en\&nrm=iso. Access on: 29 May 2016.

Revisão gramatical realizada por:

Kathia Maria Miranda.

E-mail: kmiranda54@gmail.com. 